\title{
Enhancement of Contamination Growth and Damage by Absorption Centers under UV Irradiation
}

\author{
I. Balasa ${ }^{* a}$, M. Hippler ${ }^{b}$, H. Schröder ${ }^{b}$, L. Jensen ${ }^{a}$, M. Gauch ${ }^{a}$, D. Ristau ${ }^{a}$, W. Riede ${ }^{b}$ \\ ${ }^{a}$ Laser Zentrum Hannover e.V., Laser Components Department, Hollerithallee 8, 30419 Hannover, \\ Germany; ${ }^{b}$ Deutsches Zentrum für Luft- und Raumfahrt, Institut für Technische Physik, \\ Pfaffenwaldring 38-40, 70569 Stuttgart, Germany
}

\begin{abstract}
Contamination plays a major role in lifetime of vacuum optics. Several efforts have been made to derive suitable models for lifetime prediction in laser-induced contamination related optical breakdown. But the broad spectrum of potential contaminants present in the various applications with their very specific contamination mechanisms complicates the derivation of universal optics degradation models.

As one possible contamination initiation process, the impact of optical absorption on the laser-induced contamination and resulting optical breakdown is studied in this work. A set of specifically prepared samples using nanometer sized gold particles embedded in dense IBS anti-reflecting coatings is exposed to radiation of $355 \mathrm{~nm}$ in low pressure naphthalene atmosphere. Even though the artificial defects are not in direct contact with the contaminant, their influence on the long-term optics performance in dependence on the particle concentration in the coating is evident. In the presence of naphthalene, the artificial nano-defects cause a significantly accelerated degradation compared to reference samples without those defects or in absence of the contaminant. For this specific type of contaminant, a correlation of the optical absorption and long-term durability is derived.
\end{abstract}

Keywords: laser-induced contamination, laser damage, UV, nano-defects, absorption, space-borne, optics lifetime

\section{INTRODUCTION}

Within the past decade significant improvements towards laser systems for the ultraviolet spectral (UV) region could be achieved. The demand for coherent radiation sources in shorter wavelength domains is rising not only in industrial application for production, material processing, and quality assurance. Especially in the field of atmospheric research and extraterrestrial ground-based exploitation missions, high power space-borne laser systems in the UV spectral range gained importance throughout the last decade. As the UV laser irradiation related contamination of optical components under vacuum conditions is a very critical issue, several approaches have been considered to derive predictive models for optics lifetime, being of utmost importance for the assessment of mission lifetimes.

Typically, optical components for UV applications operated under vacuum conditions in the presence of contamination show less contaminant deposition at higher sample temperatures ${ }^{1}$. Nevertheless, in most cases not the contaminant precursor molecules are found on the surface, but modified carbonaceous deposits indicating complex chemical reactions taking place on the surface of the component. Photo-induced excitation and dissociation of the precursor molecules will surely be one channel of deposition initiation. But similarly, local heating near the surface with temperatures exceeding the ones for contamination prevention can initiate chemical reactions of the contaminant precursor molecules. We assume a defect, which is for reasons of simplicity cylindrical with radius and thickness $\mathrm{r}$, specific heat capacity $\mathrm{c}_{\mathrm{p}}$, and mass density $\rho$. Its total heat capacity will therefore be

$$
C=\rho \cdot \pi \cdot r^{3} \cdot c_{p}
$$

*i.balasa@1zh.de; phone +49 5112788 476; fax +49 5112788 100; www.lzh.de 
The amount of energy that is absorbed by the defect can be estimated to be

$$
E=H \cdot \pi \cdot r^{2} \cdot\left(1-e^{-\alpha r}\right) \cdot(1-R-S),
$$

with the irradiation fluence $H$, the optical absorption coefficient $\alpha$, the reflectivity $\mathrm{R}$ in air, and the scattering loss $\mathrm{S}$. To estimate the scattering losses Rayleigh- and Mie-theory has to be applied, and especially for very small particle sizes finite size effects ${ }^{2}$ have to be considered as well. Since only the order of magnitude of the defect's temperature increase due to absorption shall be estimated, scattering losses will be neglected. This simplification will not change the order of magnitude of the calculated results. Consequently, particle dimensions for which Rayleigh theory applies shall be considered, meaning a range between $10 \mathrm{~nm}$ to $50 \mathrm{~nm}$ for an irradiation wavelength of $355 \mathrm{~nm}$. Thus, assuming a very short time period during which we can neglect thermal convection, the absorbed energy $\mathrm{E}$ is in principle capable of elevating the defect's temperature by

$$
\Delta \vartheta=\frac{E}{C} \approx \frac{H \cdot\left(1-e^{-\alpha r}\right) \cdot(1-R)}{\rho \cdot r \cdot c_{p}}
$$

Fig. 1 shows a calculation of the theoretical maximum temperature rise in dependence on the defect size for different defect materials. In the designated particle size regime the amount of absorbed energy at $355 \mathrm{~nm}$, with an irradiation fluence of $100 \mathrm{~mJ} / \mathrm{cm}^{2}$ is in principle enough to locally increase the temperature of the defect by several thousands of Kelvins, which is equivalent to melting, and especially for small particles, to evaporating them. Of course this is only a rough estimation, because the temperature of the nanoparticles will drop quickly due to heat conduction of the layer in which they are embedded in. Also the assumed scattering and reflectivity losses are only rough estimates, as they will not be valid for particles embedded in dielectric films. But the order of magnitude of the temperature rise allows to locally reach temperatures clearly above the usual bake-out regime, only induced by optical absorption. Especially nearsurface defects have the capability of initiating chemical reactions of contaminant molecules to form modified carbonaceous deposits on optical surfaces. In particular this is the critical issue in the formation of laser-induced contamination. According to Becker et. al. $^{3}$ after the formation of the first contaminant monolayer, the local temperature increases dramatically in the irradiated spot due to optical absorption by carbonaceous deposits. This increase to several hundreds of Kelvins then initiates the avalanche-like degradation of the optical component.

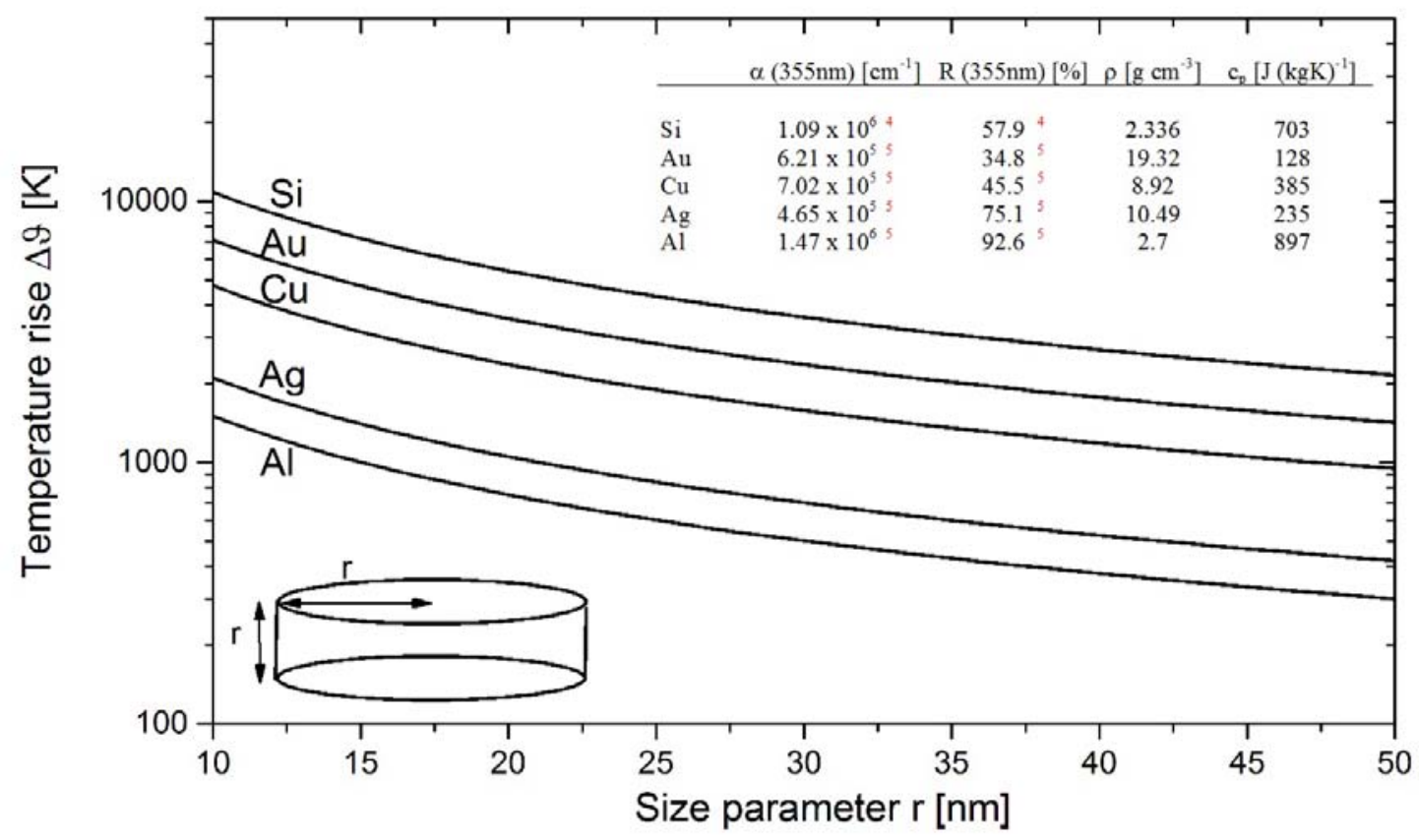

Figure 1: Calculated maximum temperature increase of nanometer sized cylindrical metallic defects in air due to absorption of laser radiation at $355 \mathrm{~nm}$ with an irradiation fluence of $100 \mathrm{~mJ} / \mathrm{cm}^{2}$. 


\section{EMBEDDING NANOPARTICLES IN ANTIREFLECTIVE COATINGS}

To study the influence of optical absorption on the formation of laser-induced contamination in the UV spectral region, coatings with artificial, absorptive defects were manufactured. The $355 \mathrm{~nm}$ antireflective (AR) coatings of interest have typical physical thicknesses below $100 \mathrm{~nm}$, as a bilayer design can already efficiently suppress reflectivity. To avoid non-linear effects due to microlensing by nodules with dimensions in the order of the application wavelength, the dimensions of the artificial defects need to be kept well below the physical thickness of the layer stack. Instantly, it becomes clear, that the seed size of the defects have to be in the range of a very few tens on nanometers in maximum. Consequently, this dimensional restriction requires a homogeneous distribution of the defects, to be able to draw distinctive conclusions even with laterally integral measurements techniques.

The defects were produced by a nanoparticle source in combination with a quadrupole mass filter from Mantis Deposition $\mathrm{GmbH}$. The magnetron sputtered target atoms agglomerate in an argon purged condensation zone to nanoclusters. The size distribution can be tuned to some extent by the length of the condensation zone and the partial pressure of the purge gas. For a more precise adjustment of the emitted nanoparticle size distribution, the quadrupole mass filter is used. Using a gold target, the source emitted nanoparticles with a principal size spectrum between 2 nanometers to 20 nanometers. All nanoparticle coatings were performed with a quadrupole mass filter set to an equivalent diameter range between $2.5 \mathrm{~nm}-7 \mathrm{~nm}$.
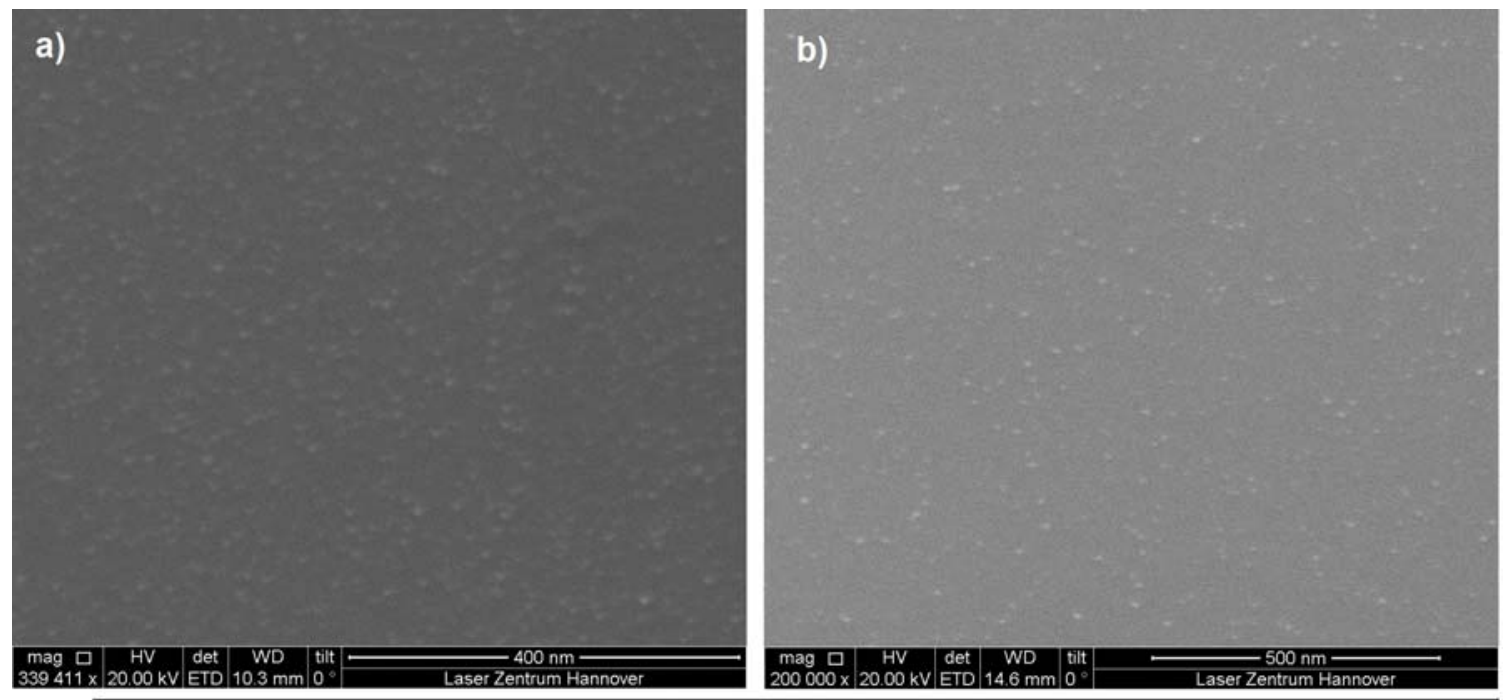

c)

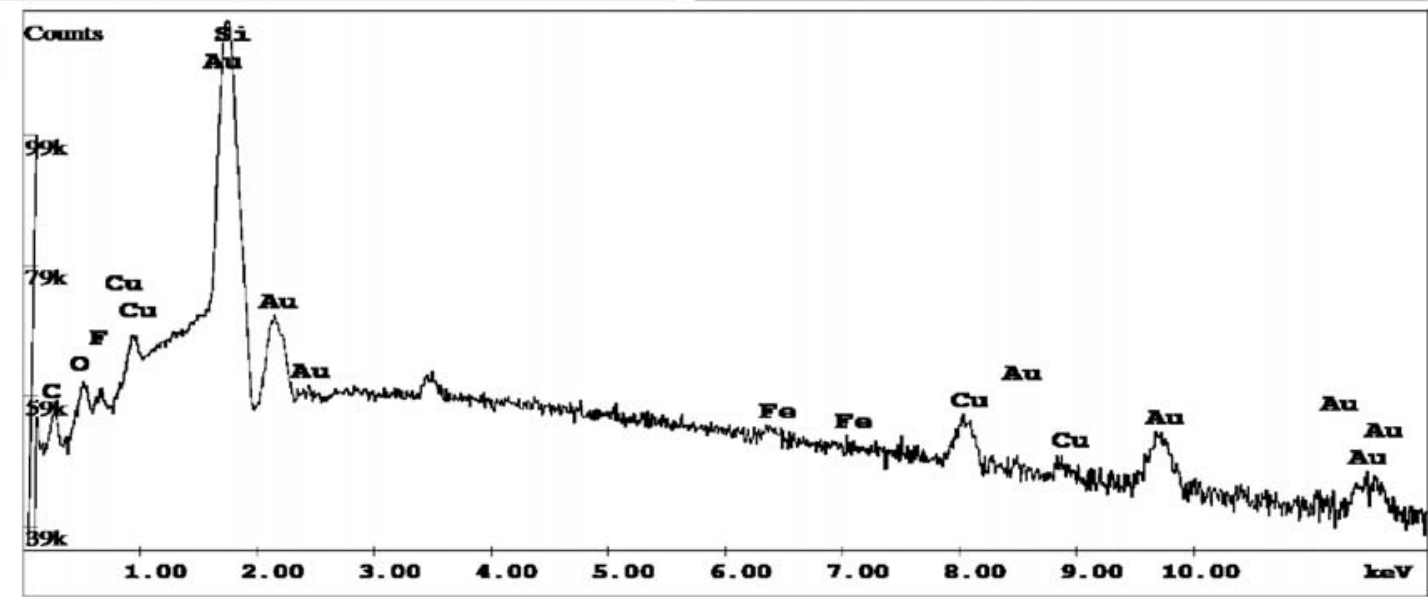

Figure 2: Scanning electron microscope (SEM) images of the silicon wafer reference substrates coated with high (a) and low (b) gold nanoparticle concentration. Energy-dispersive X-ray (EDX) spectrum of a nanoparticle multilayer produced with longer deposition time (c). The operation conditions of the nanoparticle source were maintained for both concentrations and the multilayer coating. 
To maintain the emitted size distribution of the nanoparticles, the operation conditions of the nanoparticle source were kept as constant as possible, whereas the total coating time of the substrates was varied to implement two different concentrations of nanoparticles in the sample set. The particles with size of a few nanometers are very close to the resolution limit of the scanning electron microscope (SEM). Since the fused silica substrates do not allow operating the SEM in high vacuum mode due to charging effects, reference substrates of silicon with higher electrical conductivity were introduced for characterization of the nanoparticle distributions by SEM. The results are shown in fig.2a and $2 \mathrm{~b}$. Because of the small particle size the sub mono layers of gold on silicon wafers could not be confirmed by Energydispersive X-ray spectroscopy (EDX). Instead, the composition of the particles was verified indirectly by a multilayer of nanoparticles, which was deposited on a silicon wafer using a much longer deposition time. The results in fig.2c clearly confirm the presence of gold in the multilayer. A small fraction of copper is observed as well, which may originate from the heat sink of the gold target.

The SEM images were processed (fig.3) to determine the equivalent size distribution and concentration of the gold particles. In the histograms in fig. $3 \mathrm{c}$ and $3 \mathrm{~d}$ it is obvious, that a considerable amount of particles is larger than $7 \mathrm{~nm}$ for both, high and low concentration. As the deposition is a statistical process, it is likely that the particles agglomerate on the substrate surface after deposition. Equivalent particle diameters of above $20 \mathrm{~nm}$ are observed only very rarely on both samples.

The substrates covered with nanoparticles were coated with a modified AR coating according to fig. 4a. The standard two-layer $\mathrm{HfO}_{2}-\mathrm{SiO}_{2}$ design has a physical thickness of about $95 \mathrm{~nm}$.

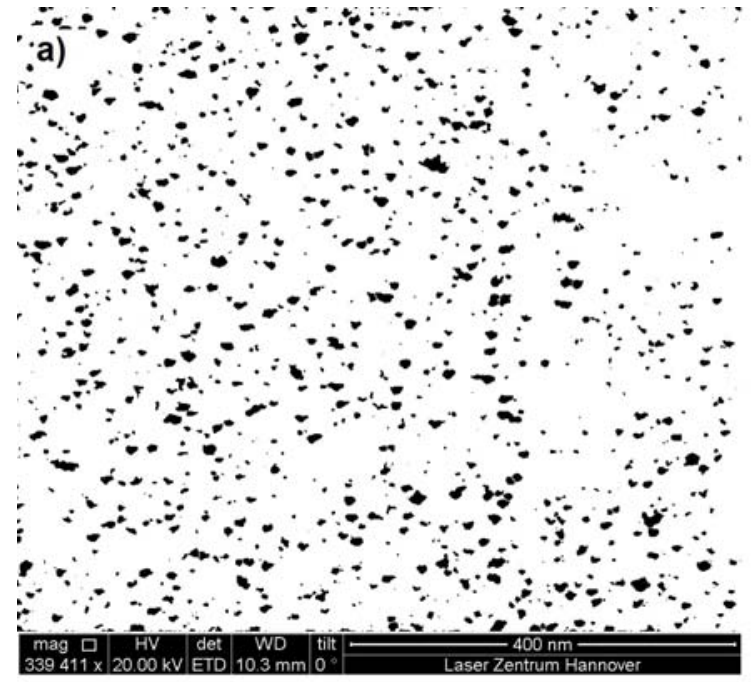

c)

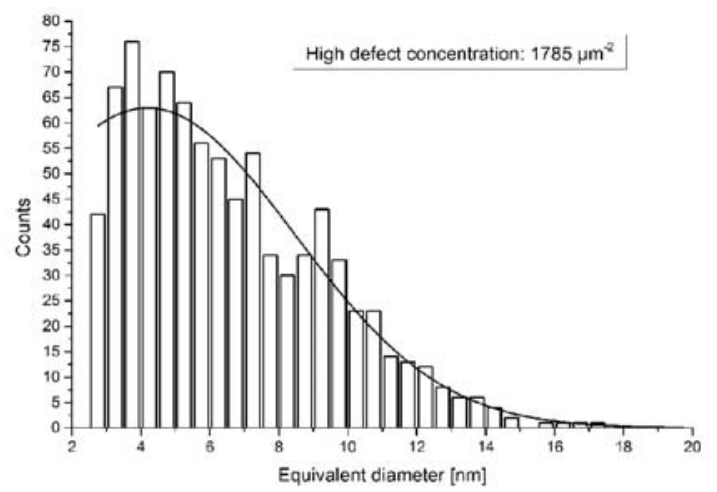

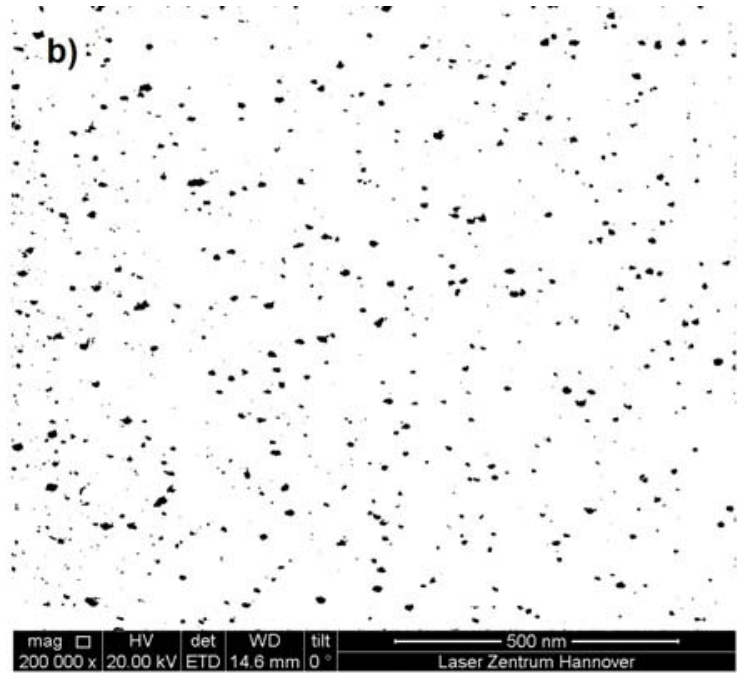

d)

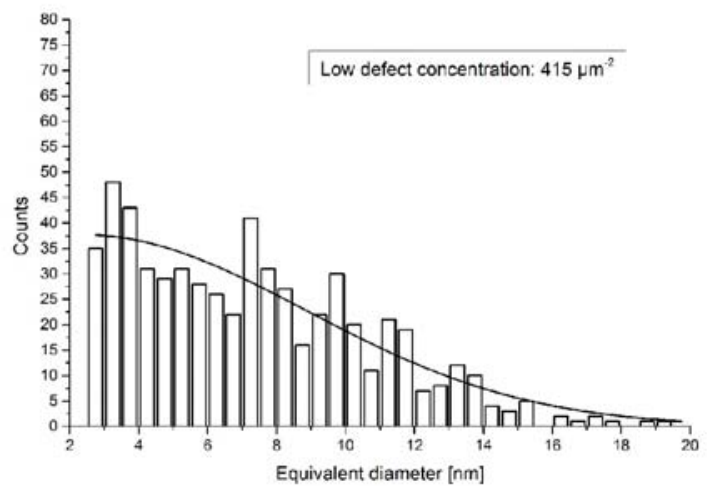

Figure 3: Analysis of SEM images from fig.1 for the high (a) and low (b) defect concentration coating. The histograms in (c) and (d) show very Gaussian like (solid lines) size distributions of the nano-defects. 
To compensate the nanoparticle dominated surface topography of the substrate to some extent, an optically ineffective additional layer of $\mathrm{SiO}_{2}$ with an optical thickness of $\lambda / 2$ at $355 \mathrm{~nm}$ (HWOT) was introduced between substrate and AR coating. The total layer stack thickness was therefore increased to $205 \mathrm{~nm}$. This layer stack was deposited on both sides of the fused silica substrates, whereas gold nanoparticles were deposited on only one side of each substrate. By this means, each AR-coated sample is its own reference at the same time.
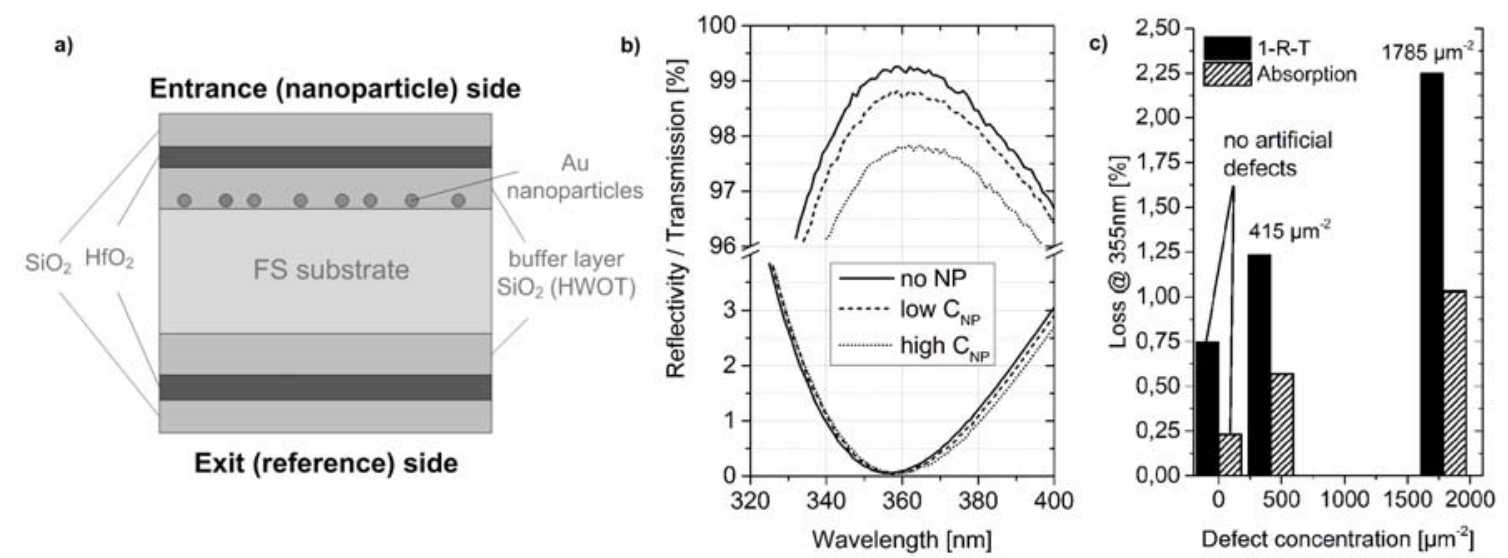

Figure 4: Schematic layout of the artificial defect samples (a), UV transmission and reflectivity spectra (b), and calculated total optical loss compared to measured absorption at $355 \mathrm{~nm}$ (c) for the two defect concentrations and a reference sample with same both-side antireflective coating but without any artificial defects.

For each artificial defect sample, a reference sample without artificial particles but with the same layer stack on both sides was produced. All front coatings were deposited in one coating run, the back side coatings in a second deposition run. Fig. $4 \mathrm{~b}$ and $4 \mathrm{c}$ show the total optical losses and absorption losses at $355 \mathrm{~nm}$. The reference samples have a rather high absorption of about $0.22 \%$. For not affecting the observed nano-defects, the samples were not annealed, which is the reason for the rather high absorption due to oxygen deficiencies in the coating. Nevertheless, an even more significant increase in absorption is observed for the samples with incorporated defects. The low concentration samples show an increase to above $0.55 \%$, the high concentration samples even up to $1 \%$. Subtracting the offset absorption of $0.22 \%$, an increase of a factor of 2.3 in absorption is observed for an increase in the concentration by a factor of 4.3 .

\section{LASER-INDUCED CONTAMINATION TEST BENCH}

The setup for LIC tests is shown in fig. 5 and described in more detail in reference 6. Pumping is performed by a turbo molecular and an oilfree scroll pump. Pirani, Penning and capacitance manometers are installed for pressure sensing. The composition and partial pressure of contamination material is determined by a residual gas analayzer (RGA). The contaminant is supplied by a small contamination chamber which is connected via a needle valve to the main chamber. The sample holder allows testing 3 different optics simultaneously under the same condition. A long distance microscope with a resolution of $1 \mu \mathrm{m} /$ pixel monitors the laser-induced fluorescence and allows in-situ investigation of damage onset. The transmission of the optics is monitored in-situ. The laser source is a pulsed Nd:YAG laser with frequency tripling, a pulse duration of $10 \mathrm{~ns}$, and a repetition frequency of $1 \mathrm{kHz}$. The UV beam has a Gaussian profile with a 1/( $\mathrm{e}^{2}$-diameter of $300 \mu \mathrm{m}$. During laser irradiation, the chamber windows are heated to $150^{\circ} \mathrm{C}$ to reduce laser-induced contamination. Naphthalene is used as contamination material in the tests. By adjustment of the needle valve and the pumping speed a constant contamination pressure in the range of $1 \times 10^{-5} \mathrm{mbar}$ is achieved. The laser fluence is varied between $120 \mathrm{~mJ} / \mathrm{cm}^{2}$ and $800 \mathrm{~mJ} / \mathrm{cm}^{2}$. For validation of chamber cleanliness a blank test without any contamination material is performed prior to the LIC tests. 


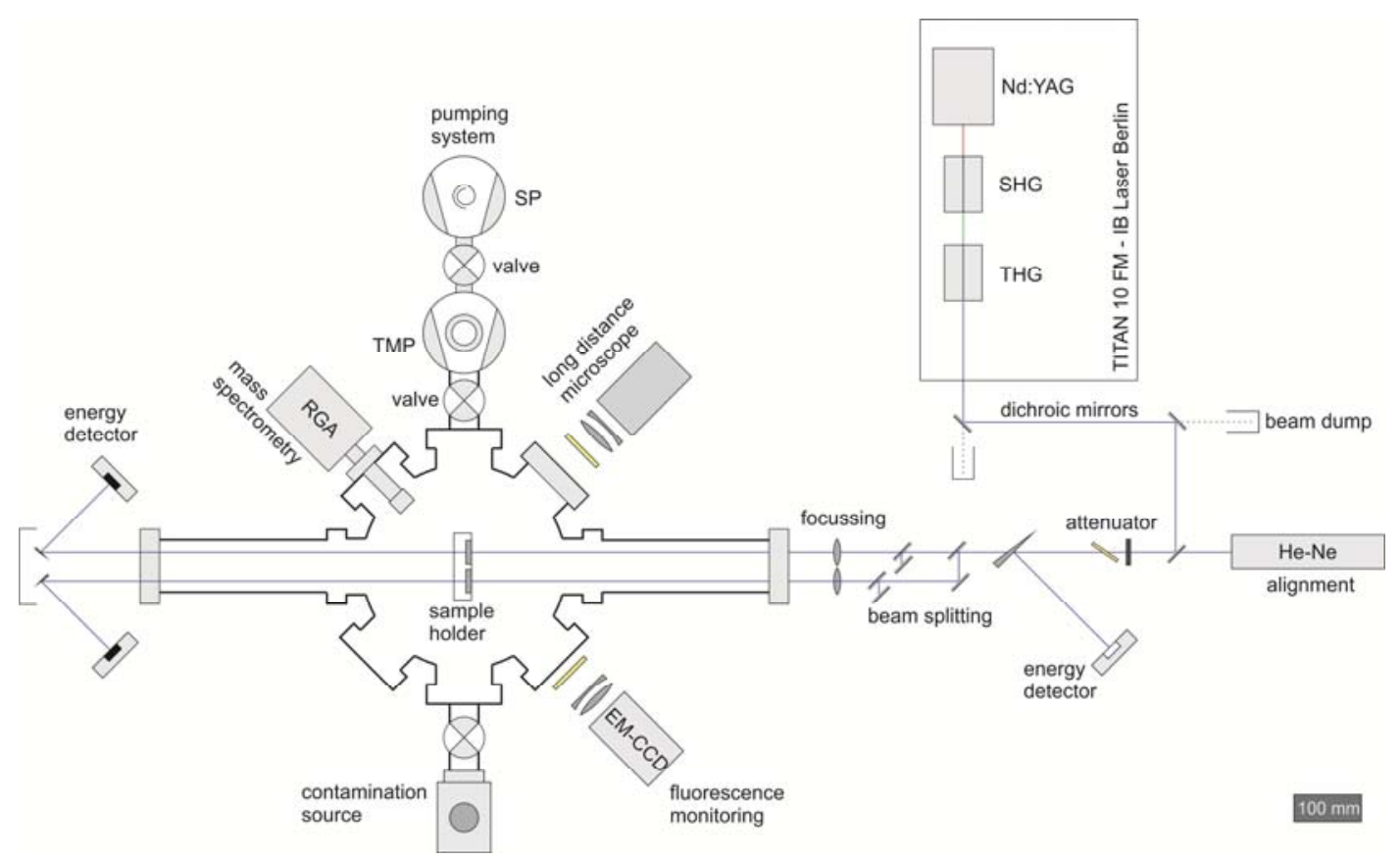

Figure 5: Ultra-high vacuum laser-induced contamination (LIC) test bench operated at a laser wavelength of $355 \mathrm{~nm}$ and a repetition frequency of $1 \mathrm{kHz}^{6}$. The initial laser beam is splitted into four beams of equal power to be able to test 3 different samples simultaneously and to have one reference for the determination of the in-situ transmission.

\section{RESULTS}

\subsection{LIC blank test}

To clearly identify the contaminant naphthalene as the driving force of the optics degradation a blank test was performed. For the observation of any unwanted residues in the test chamber that cause optics contamination and/or degradation, the irradiation fluence was set to $790 \mathrm{~mJ} / \mathrm{cm}^{2}$, which is according to previous experiments ${ }^{6}$ clearly above the contamination induced damage threshold. During 60 minutes with a repetition frequency of $1 \mathrm{kHz}$ at a pressure of $7 \times 10^{-9}$ mbar no in-situ transmission degradation of either low- or high defect concentration sample, nor reference sample was observed within the transmission measurement accuracy (fig. 6). Differential Interference Contrast (DIC) microscopy of the front- and back sides of all three test samples revealed no visible changes of the exposed test sites. According to these results, the findings in the following sections can be attributed to the presence of naphthalene in the test chamber.

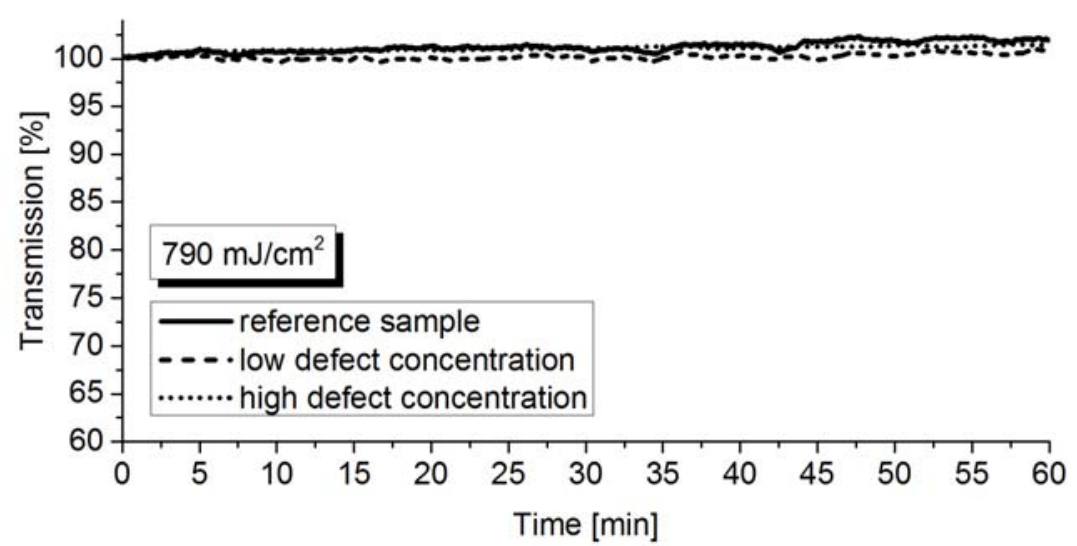

Figure 6: In-situ transmission of reference, low- and high nanoparticle concentration samples during blank test at an irradiation fluence of $790 \mathrm{~mJ} / \mathrm{cm}^{2}$ and a pressure of $7 \times 10^{-9}$ mbar. No degradation of the transmission is observed in absence of naphthalene. 


\subsection{LIC tests at $120 \mathrm{~mJ} / \mathrm{cm}^{2}$}

Two tests with an irradiation fluence of $120 \mathrm{~mJ} / \mathrm{cm}^{2}$ were carried out with durations of 60 minutes (fig. 7) and 120 minutes (fig. 8). In both tests, the degradation of the reference sample and the sample with low defect concentration is very similar, whereas the sample with high concentration of nanoparticles showed significant degradation in transmission. In terms of in-situ transmission, the second experiment occurs to be the continuation of the first test with shorter duration (compare fig. 7a and 8a). The damage/contamination patterns of the exposed sites tend to indicate a similar contamination mechanism, as the pancake-like growth after 60 minutes shown in fig. 7c and 7d is followed by ablation of the deposit beginning in the center (doughnut-like profile) which is shown in fig. 8c. The carbonaceous nature of the deposits is verified by the EDX analysis in fig. 9, where especially at the edges of the doughnut-structure significant amounts of carbon are detected. These results mainly confirm the growth of deposits in the range of a few micrometers on the sample surface as shown for example in fig. $8 \mathrm{c}$.
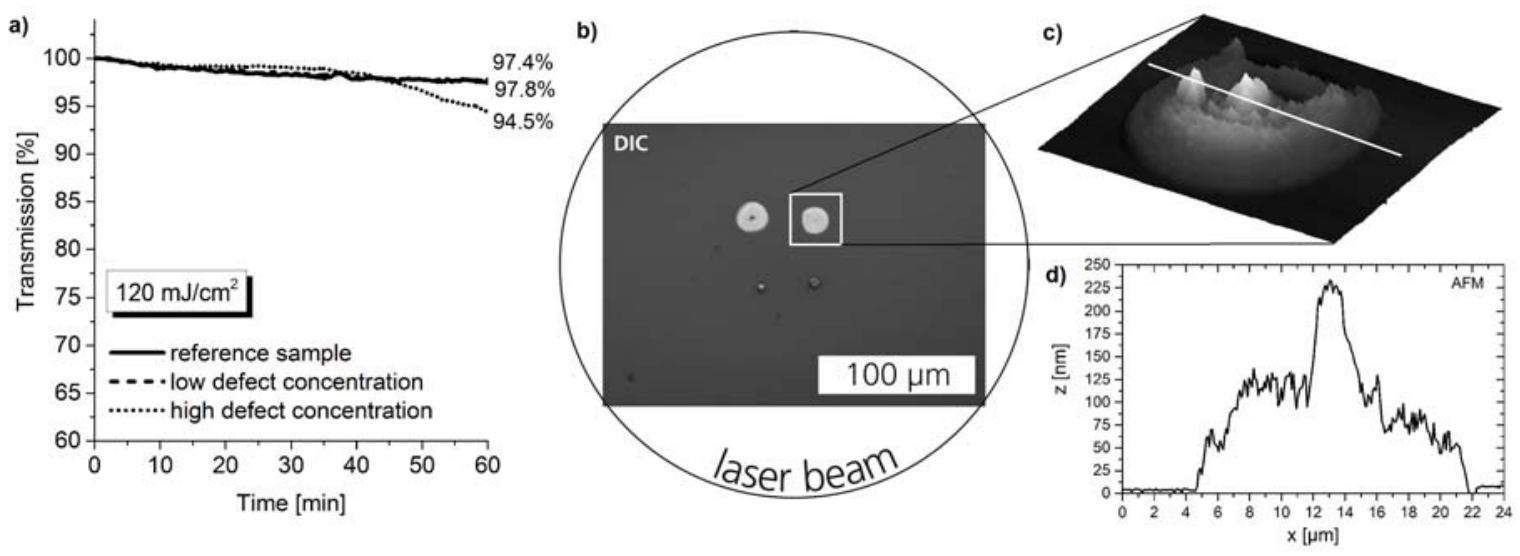

Figure 7: In-situ transmission during laser-induced contamination tests with a naphthalene partial pressure of $1 \times 10^{-5}$ mbar and an irradiation fluence of $120 \mathrm{~mJ} / \mathrm{cm}^{2}$ for a total test duration of 60 minutes (a). The dimensions of the laser beam profile are illustrated in (b) with respect to the ex-situ DIC microscopy images of the deposits found on the high defect concentration sample. The topography of the deposit was recorded by AFM and is shown in (c) together with a representative line scan (d).
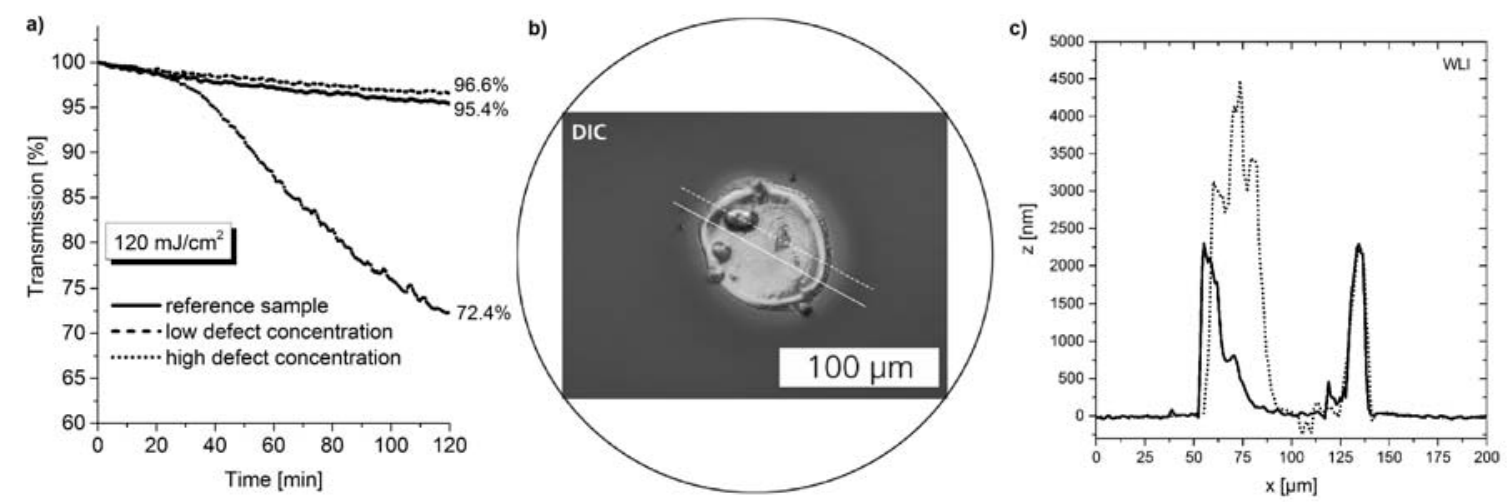

Figure 8: In-situ transmission during laser-induced contamination tests with a naphthalene partial pressure of 1 x $10^{-5}$ mbar and an irradiation fluence of $120 \mathrm{~mJ} / \mathrm{cm}^{2}$ for a total test duration of 120 minutes (a). The dimensions of the laser beam profile are illustrated in (b) with respect to the ex-situ DIC microscopy images of the deposits found on the high defect concentration sample together with representative line scans (c).

As illustrated in fig. $7 \mathrm{~b}$ and $8 \mathrm{~b}$, the deposit formation/ablation occurs only in the high fluence region of the beam profile. Furthermore, fig. $7 \mathrm{~b}$ suggests the initiation of deposit growth by seeds in or on top of the coating. As the gold nanoparticles are distributed homogeneously in the coating with a spacing of a very few tens of nanometers, it is very 
unlikely that these artificial defects are the seeds themselves. The isolated seeds in fig. $7 \mathrm{~b}$ occur to initiate deposit formation, which overlap to one joint deposit in the high fluence region of the laser beam after expansion in lateral direction fig. $8 \mathrm{~b}$. There was no sign of any deposit formation, damage, or any degradation of the back sides (beam exit sides) of the samples.
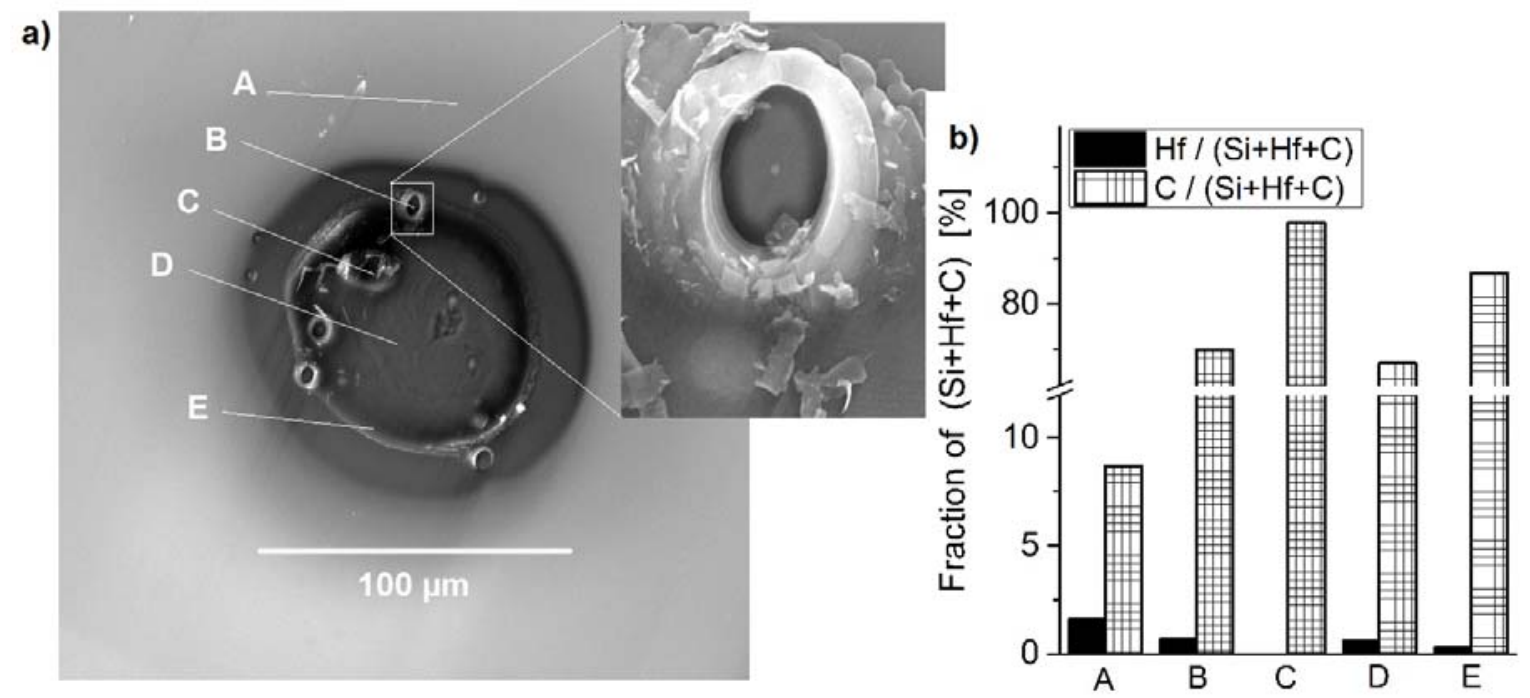

Figure 9: SEM images of the damage site at the beam entrance side of the high defect concentration sample after the test at $120 \mathrm{~mJ} / \mathrm{cm}^{2}$ (a) and corresponding elemental compositions determined by EDX (b).

\subsection{LIC tests at $240 \mathrm{~mJ} / \mathrm{cm}^{2}$}

A dramatic difference is observed at the end of the test with an irradiation fluence of $240 \mathrm{~mJ} / \mathrm{cm}^{2}$. Once again, the reference sample and the low defect concentration sample show nearly identical behavior, whereas the transmission of the high concentration sample drops down $62.2 \%$ after an hour of exposure (fig. 10a). The depth profiles recorded via WLI (fig. 10c) show ablation of the coating down to a few micrometers into the substrate. According to the results of the EDX analysis shown in fig. 11b the fraction of Hafnium found in the crater decreases with crater depth. But even in the sub-crater structure residues of Hafnium in the order of a fifth of the initial fraction can be found. The EDX results show as well, that besides material ablation, and even though no carbonaceous growth is found at the main damage site, a significant amount of carbon is incorporated in the crater structure.
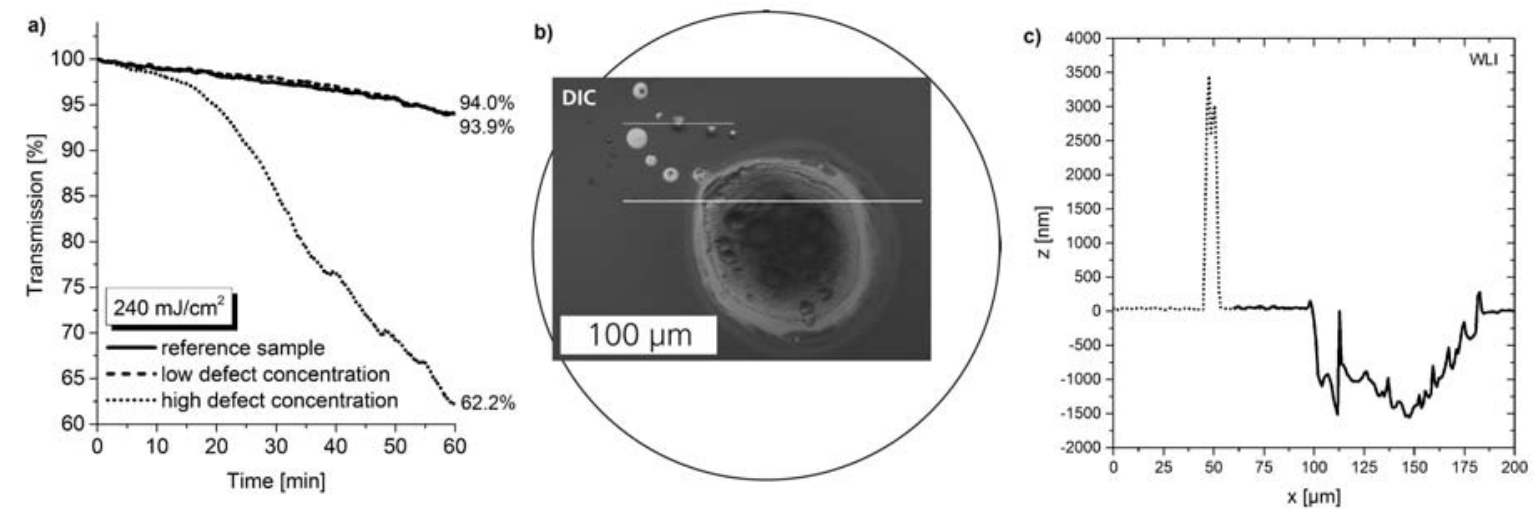

Figure 10: In-situ transmission during laser-induced contamination tests with a naphthalene partial pressure of $1 \times 10^{-5}$ mbar and an irradiation fluence of $240 \mathrm{~mJ} / \mathrm{cm}^{2}$ for a total test duration of 60 minutes (a). The dimensions of the laser beam profile are illustrated in (b) with respect to the ex-situ DIC microscopy images of the deposits found on the high defect concentration sample together with representative line scans (c). 

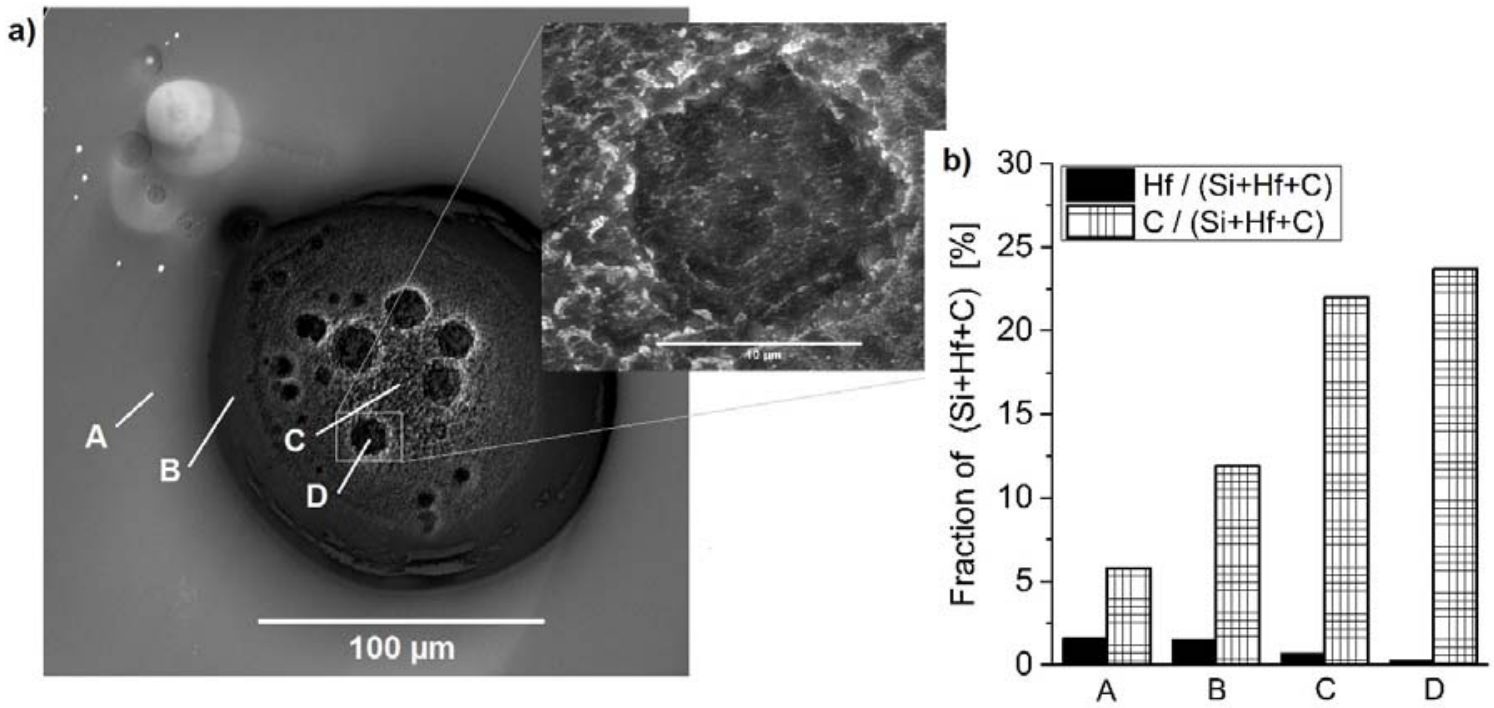

Figure 11: SEM images of the damage site at the beam entrance side of the high defect concentration sample after the test at $240 \mathrm{~mJ} / \mathrm{cm}^{2}$ (a) and corresponding elemental compositions determined by EDX (b).

a)

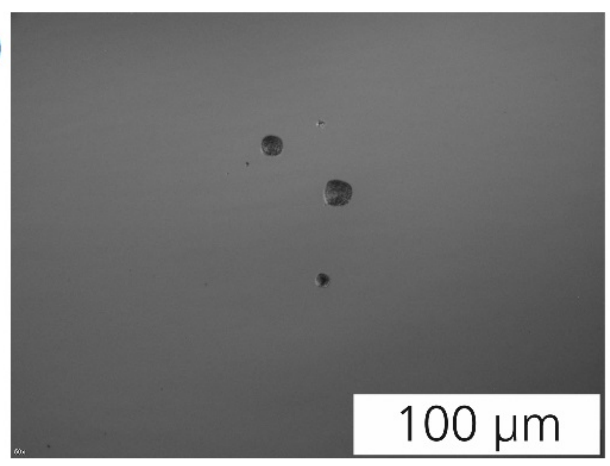

b)

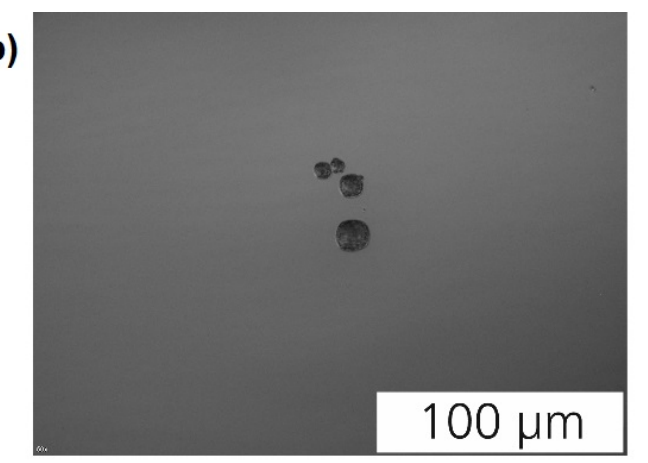

c)

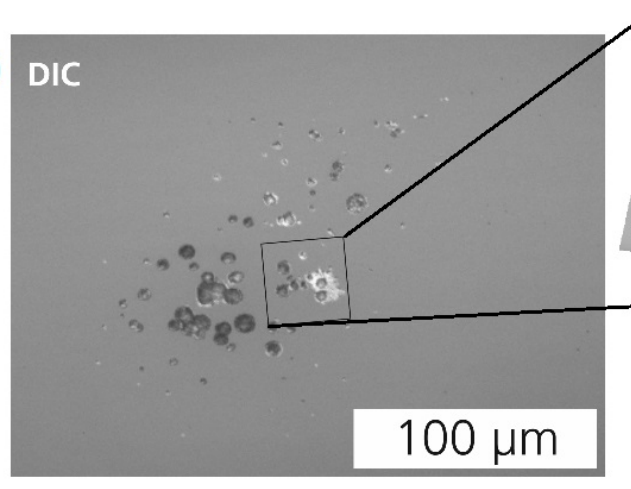

d)

\section{AFM}

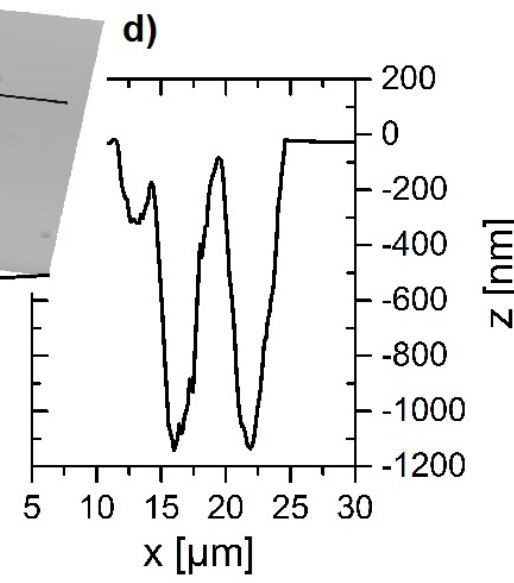

Figure 12: Ex-situ DIC and AFM images of the beam exit side (no artificial defects embedded in AR coating) of reference sample (a), low defect concentration sample (b) and high defect concentration sample (c and d) after 60 minutes of exposure at $240 \mathrm{~mJ} / \mathrm{cm}^{2}$ in naphthalene atmosphere with a partial pressure of $1 \times 10^{-5} \mathrm{mbar}$. 
In contrast to the tests at $120 \mathrm{~mJ} / \mathrm{cm}^{2}$, the rear sides of all three samples show very similar micro-channel formations with depths of a few micrometers (fig. 12). The morphology of the defects resembles the patterns observed for substrates and dense coatings in reference 6 at similar irradiation fluence. Again the damage threshold is at least one order of magnitude lower than typical values for $\mathrm{HfO}_{2} / \mathrm{SiO}_{2}$ coatings for the UV spectral range in absence of naphthalene. No traces of any degradation of the beam entrance sides of reference sample and low defect concentration sample were observed by means of DIC microscopy.

\section{CONCLUSIONS}

Gold nanoparticles were embedded in dense $\mathrm{HfO}_{2} / \mathrm{SiO}_{2} \mathrm{AR}$ coatings by IBS deposition technique. Even though a dense layer system of about $200 \mathrm{~nm}$ of physical thickness separated the artificial nano-defects from the naphthalene molecules, a strong influence of the high defect concentration samples on the laser-induced contamination and contamination induced damage could be observed well below common laser-induced damage thresholds in absence of any contamination. In dependence on irradiation fluence and exposure time, an initial deposit formation on the optics surface was observed, which was again ablated with longer exposure time. For higher irradiation fluence even coating ablation occurred. On the reference sides of the samples, where only an identical AR coating was deposited without any nanoparticles, at higher fluence the formation of micro-channels was observed in good agreement to previous tests ${ }^{6}$. Even though the high- and low defect concentration samples differed only by a factor of 2 with respect to total optical loss as well as absorption at $355 \mathrm{~nm}$, the low defect concentration sample showed now major deviation in terms of degradation compared to the reference samples without any nanoparticles. It is unclear whether the short test duration or a probable threshold behavior is responsible for this result. The striking difference in contamination and damage behavior, as well as in the damage morphologies of beam entrance- and beam exit side of the high defect concentration sample indicates a significant influence of the artificial absorption centers on the performance of the coating.

\section{ACKNOWLEDGMENT}

The authors thank the European Space Agency for the financial support within the framework of "Optical Components Materials and Process Development and Validation for High Power Space Borne Lasers" (Contract No. 4000102602/11/NL/CP).

\section{REFERENCES}

[1] Riede, W., Schröder, H., Bataviciute, G., Wernham, D., Tighe, A., Pettazzi, F., Alves, J., "Laser-induced contamination on space optics," Proc. of SPIE 8190, 81901E (2011)

[2] Myroshnychenko, V., Rodriguez-Fernandez, J., Pastoriza-Santos, I., Funston, A.M., Novo, C., Mulvaney, P., Liz-Marzan, L.M., Garcia de Abajo, F.J., "Modeling the optical response of nanoparticles," Chem. Soc. Rev. 37, 1792-1805 (2008)

[3] Becker, S., Pereira, A., Bouchut, P., Geffraye, F., Anglade, C., "Laser-induced contamination of silica coatings in vacuum," Proc. of SPIE 6403, 64030J (2006)

[4] Rakić, A. D., Djurišic, A. B., Elazar, J. M., Majewski, M. L., "Optical properties of metallic films for verticalcavity optoelectronic devices,” Appl. Opt. 37 (22), 5271-5283 (1998)

[5] Vuye G., Fisson, S., Nguyen Van, V., Wang, Y., Rivoty, J., Abeles, F., "Temperature dependence of the dielectric function of silicon using in situ spectroscopic ellipsometry," Thin Solid Films 233, 166-170 (1993)

[6] Schröder, H., Wagner, P., Kokkinos, D., Riede, W., Tighe, A., "Laser-induced contamination and its impact on laser damage threshold," Proc. of SPIE 8885, 88850R (2013) 